\title{
Editorial
}

\section{Reflexiones sobre la vasectomía sin bisturí}

\author{
Reflections on The No-scalpel Vasectomy \\ Reflexões sobre a vasectomia sem bisturi
}

Judith del Carmen Jiménez-Hernández, ${ }^{*}$ Patricia Flores-Huitrón, ${ }^{* *}$ Efrén Raúl Ponce-Rosas ***

La vasectomía sin bisturí (vSB) es un método anticonceptivo permanente, sencillo de realizar y que requiere únicamente de dos instrumentos especialmente diseñados para el procedimiento, en el cual, previa anestesia local y mediante punción de una pinza especial, se aíslan los conductos deferentes, se ligan y cortan sin que sea necesario el uso del bisturí. ${ }^{1}$ En México, la Secretaría de Salud federal promueve la vsB como el método permanente de planificación familiar para el hombre, que consiste en la interrupción del paso de los espermatozoides, mediante el corte u oclusión de los conductos deferentes. ${ }^{2}$

La vsB fue desarrollada en 1974 por el doctor Li Shunqiang en el Instituto Chongqing de Investigaciones Científicas en Planificación Familiar, de la provincia de Sichuan, China; ${ }^{2}$ posteriormente, la técnica quirúrgica fue introducida en la comunidad médica occidental en 1985 por el doctor Marc Goldstein del Cornell Medical Center-New York Hospital. En México, la vsB fue introducida en 1989 en el Instituto Mexicano del Seguro Social (IMSS); en 1993, la Dirección General de Planificación Familiar (actualmente Centro Nacional de Equidad de Género y Salud Reproductiva) de la Secretaría de Salud inició el programa de vsB en el primer nivel de atención con cobertura nacional. ${ }^{2}$
En 2012, la Asociación Americana de Urología consideraba la vsB como el procedimiento con mínima invasión quirúrgica más recomendado comparado con la vasectomía convencional (con bisturî. ${ }^{3-5}$ Stockton y su grupo de colaboradores refieren la vsB como el procedimiento más popular para el control definitivo de la fertilidad en los hombres de Estados Unidos. ${ }^{6}$

La Organización de las Naciones Unidas (ONU) publicó en su reporte de 2011 sobre anticoncepción en el mundo ${ }^{7}$ (188 países) una prevalencia global por cualquier método anticonceptivo de $62 \%$ en más de 1178 millones de mujeres de entre 15 y 49 años, casadas o unidas con pareja estable; en dicho reporte, la prevalencia de vasectomía mundial se estimó en $2.5 \%$ comparada con 3.4\% reportada por la ONU en $2005 .{ }^{8}$ En el caso de México, se estimó para 2005 una prevalencia de vasectomía de $1.3 \%{ }^{8}$ y se reportó como desconocida en el informe de la ONU de 2011 . $^{7}$

Para 2011, la vasectomía ocupó el séptimo lugar en la prevalencia de anticoncepción mundial ${ }^{7}$ con $2.5 \%$; los primeros lugares fueron para la píldora (21.4\%), el condón (17.1\%), el dispositivo intrauterino (DIU) $(12.4 \%)$, la retirada $(8 \%)$, el ritmo $(5.2 \%)$ y la salpingoclasia (3.1\%). Sin embargo, los porcentajes cambian significativamente cuando se analizan los datos por regiones (continentes), favoreciendo a los países desarrollados, en los cuales destacan los métodos quirúrgicos en mujeres, el DIU, los hormonales y el condón. En el caso de México, las cifras del reporte de la ONu de 2011 (disponibles del año 2006) fueron métodos modernos: contracepción de emergencia, condón femenino, implante, salpingoclasia y vasectomía con o sin bisturí (38.4\%) (no se distingue entre los porcentajes para

\footnotetext{
"Médico familiar, unidad de medicina familiar (UMF) no. 64, Instituto Mexicano del Seguro Social (IMSS), México. "Médico familiar, responsable del programa Vasectomía sin Bisturí en el centro de salud "Dr.José CastroVillagrana", Secretaría de Salud del Distrito Federal, México. ${ }^{* * *}$ Profesor de la Subdivisión de Medicina Familiar, División de Estudios de Posgrado, Facultad de Medicina,
}

Este artículo debe citarse: Jiménez-Hernández JC, Flores-Huitrón P, Ponce-Rosas ER. Universidad Nacional Autónoma Reflexiones sobre la vasectomía sin bisturí. Aten Fam. 2014;21(2):33-34. de México (UNAM) 
cada método), DIU (11.6\%), condón (6.4\%), inyectables ( $5 \%$ ) y píldora (4.7\%); y métodos tradicionales: ritmo, retirada, abstinencia, lactancia materna, duchas vaginales $(4.4 \%){ }^{7}$

Es interesante observar que los países desarrollados mantienen tasas de prevalencia más elevadas de cualquier método anticonceptivo en comparación con los países menos desarrollados; la prevalencia es consistentemente mayor en $10 \%$ o más, ${ }^{7}$ dato que llega a diferencias de $40 \%$ cuando se compara con los países más subdesarrollados del mundo. ${ }^{7,8}$ Lo anterior hace suponer que el perfil del varón que acepta la vasectomía posiblemente se relacione con ciertas características sociodemográficas, las cuales pueden ser utilizadas como estrategia de mercadotecnia social en salud ${ }^{9}$ para la promoción, información y consejería de este método de anticoncepción. Sobre la experiencia y el perfil de los pacientes que se realizan vsB, se han publicado estudios importantes llevados a cabo en las principales instituciones de salud de México; es el caso de Prieto y cols., quienes reportan un estudio de 10 años de experiencia en el IMSs con 395 pacientes. ${ }^{10}$ Otro trabajo lo efectuaron Lara y cols. ${ }^{11}$ en el Instituto Nacional de Perinatología, reportando la experiencia de cinco años con 596 pacientes; mientras que en el Instituto de Seguridad y Servicios Sociales para los Trabajadores del Estado (ISSSTE) se reportó la experiencia de 25 años en los que se realizó vsB en 2237 pacientes. $^{12}$

En México, la vsB es una opción más de anticoncepción quirúrgica; por su naturaleza de mínima invasión, disminuye el temor de los varones a la incisión y a la propia intervención quirúrgica. Sin embargo, puede considerarse que aún no constituye un método suficientemente utilizado, por lo que su difusión es un imperativo que no puede postergarse.

\section{Referencias}

1. Manual Técnico de Vasectomía sin Bisturí. México: Dirección General de Salud Reproductiva, Secretaría de Salud; 1999.

2. Actualización en vasectomía. México: Centro $\mathrm{Na}$ cional de Equidad de Género y Salud Reproductiva, Secretaría de Salud; 2007.

3. Sharlip ID, Belker AM, Honig S, Labrecque M, Marmar JL, Ross S, et al. Vasectomy: AUA guideline. American Urological Association, Education and Research Inc; 2012.

4. Marmar JL, Kessler S, Hartanto VH. A minimally invasive vasectomy with the no suture, inline method for vas occlusion. International Journal of Fertility and Women's Medicine. 2001;46:257.

5. Moon H. Minimally invasive vas surgery using a newly designed double-ringed clamp. World Journal of Urology. 2010;28:205.

6. Stockton MD, Kim ED. No scalpel vasectomy [Internet] [citado 2013 Jul 5]. Disponible en: http:// emedicine.medscape.com/article/148512-overview

7. United Nations, Department of Economic and Social Affairs, Population Division (2011). World Contraceptive Use 2011.

8. United Nations, Department of Economic and Social Affairs, Population Division (2005). World Contraceptive Use 2005.

9. Manual de Mercadotecnia Social en Salud. México: Dirección General de Promoción de la Salud, Dirección de Estrategias y Desarrollo de Entornos Saludables, Subdirección de Mercadotecnia Social, Secretaría de Salud; 2010.

10. Prieto ECD, Méndez CR, Medina CJL, Trujillo HB, Vásquez C. Vasectomía sin bisturí. Experiencia de 10 años. Rev Med Imss. 2004;42(4):337-41.

11. Lara RR, Velázquez RN, Reyes ME. Vasectomía sin bisturí. Perfil del usuario y resultados. Ginecol Obstet Mex. 2010;78(4):226-31.

12. Córdoba BDI, Sapién LJS, Valdepeña ER, Patiño OSP, Castellanos HH. Vasectomía: reseña de 25 años en el Centro de Cirugía Ambulatoria en el ISSSTE. Revista de Especialidades Médico Quirúrgicas. 2011;16(1):33-8. 